\title{
Paper-Based Amperometric Sensor for Acetylcholinesterase Determination Using Screen-Printed Graphene Electrode
}

\author{
Panraksa P. ${ }^{1,2}$, Siangproh W. ${ }^{3}$, Khampieng T.4, Chailapakul O. ${ }^{2,5}$, Apilux A. ${ }^{4}$ \\ 1 Program in Biotechnology, Faculty of Science, Chulalongkorn University, 254 Phayathai Road, \\ Patumwan, Bangkok, Thailand, \\ 2 Electrochemistry and Optical Spectroscopy Center of Excellence (EOSCE), Department of \\ Chemistry, Faculty of Science, Chulalongkorn University, 254 Phayathai Road, Patumwan, Bangkok, \\ Thailand, \\ ${ }^{3}$ Department of Chemistry, Faculty of Science, Srinakharinwirot University, Sukhumvit 23, Wattana, \\ Bangkok, Thailand, \\ ${ }^{4}$ Department of Clinical Chemistry, Faculty of Medical Technology, Mahidol University, 999 \\ Phutthamonthon 4 Road, Salaya, Nakhon Pathom 73170, Thailand, \\ ${ }^{5}$ Center of Excellence on Petrochemical and Materials Technology, Chulalongkorn University, \\ Patumwan, Bangkok, Thailand \\ corawon@chula.ac.th, amara.apl@mahidol.ac.th
}

\begin{abstract}
:
Acetylcholinesterase (AChE) is an enzyme in cholinergic synapse which is an indicator of pesticides toxicity. Therefore, AChE activity determination is essential. In this work, a simple, rapid, sensitive, and selective paper-based electrochemical sensor for determination of AChE was firstly developed. A screen-printed graphene electrode (SPGE) was used as a working electrode due to its excellent electrical conductivity, electron mobility, high sensitivity, and cost effectiveness. The amperometric determination of AChE is based on the electrochemical signal of thiocholine (TCh) which is generated from the hydrolysis of acetylthiocholine (ATCh) by AChE. For AChE detection, ATCh immobilized sheet was stacked onto the detection sheet using double adhesive tape. After that, AChE samples were dropped onto the back side of an ATCh immobilized sheet and incubate for only $60 \mathrm{~s}$. Then the oxidation current was generated from TCh which can be measured by amperometric method. The interferences of $A C h E$ determination, which is glutathione (GSH), can be avoided by applying the potential of $0.5 \mathrm{~V}$ vs. $\mathrm{Ag} / \mathrm{AgCl}$ onto the SPGE and the oxidation current which related to $\mathrm{AChE}$ concentration was measured. Under optimal conditions, a linear range of the developed sensor in the range of 0.1 to $15 \mathrm{U} / \mathrm{mL}$ and the limit of detection (LOD) of $0.1 \mathrm{U} / \mathrm{mL}$ were obtained. Additionally, the proposed sensor was successfully applied for the AChE determination in blood samples and the results were in good agreement with a spectrophotometric method which is conventional method.
\end{abstract}

Key words: paper-based sensor, screen-printed graphene electrode, acetylcholinesterase, glutathione, amperometric method 